
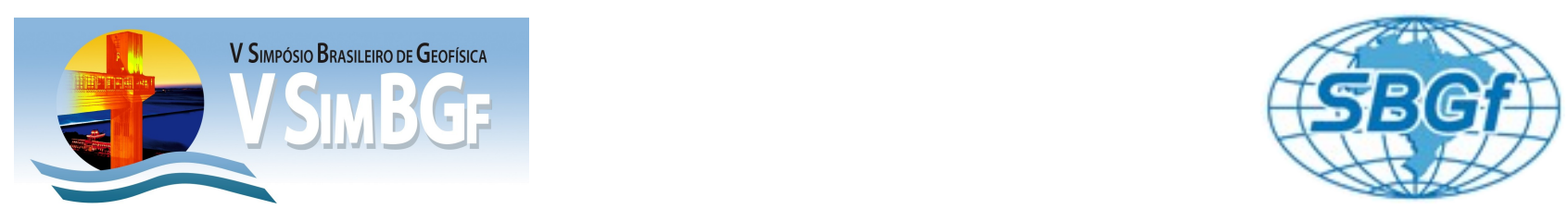

\title{
Magnetômetro fluxgate para trabalhos de campo
}

Luiz Benyosef* - benyosef@on.br

André Wiermann

José Roberto Lopes de Carvalho

Observatório Nacional

Copyright 2012, SBGf - Sociedade Brasileira de Geofísica

Este texto foi preparado para a apresentação no V Simpósio Brasileiro de Geofísica, Salvador, 27 a 29 de novembro de 2012. Seu conteúdo foi revisado pelo Comitê Técnico do V SimBGf, mas não necessariamente representa a opinião da SBGf ou de seus associados. É proibida a reprodução total ou parcial deste material para propósitos comerciais sem prévia autorização da SBGf.

\section{Abstract}

A fluxgate magnetometer has been developed at LDSM/ON in order to support field surveys and geological prospection. Its triaxial sensor was built using a CoFeSiB based alloy, in toroidal shape, operating in fields up to $\pm 50.000 \mathrm{nT}$, showing a resolution of $0.1 \mathrm{nT}$, and $47.4 \mathrm{pTrms}$ of total noise, within a frequency band of $0.0-2.0 \mathrm{~Hz}$.

\section{Introdução}

O Laboratório de Desenvolvimento de Sensores Magnéticos do Observatório Nacional (LDSM/ON) vem desenvolvendo e construindo sensores e magnetômetros baseados em diferentes tecnologias, em sua maioria baseados no princípio fluxgate. Estes sensores são de alta resolução, desenvolvidos com materiais magnéticos amorfos de base CoFeSiB ou nanocristalinos (FeSiCuNbBe) e, de acordo com a aplicação, podem ser construídos com diferentes geometrias e dimensões.

$\mathrm{Na}$ busca por instrumentos cada vez mais sensíveis e com maior gama de aplicações, o LDSM/ON tem investido na melhoria nos circuitos de tratamento do sinal fluxgate, assim como na sua conversão digital e no subsequente processamento numérico dos dados.

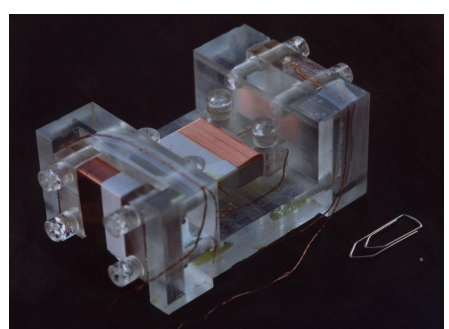

Figura 1 - Sensor fluxgate, com material magnético amorfo CoFeSiB desenvolvido no LDSM/ON.

Para a construção do instrumento descrito neste trabalho, foi utilizado um sensor baseado em material amorfo, que produz o menor ruído obtido até o presente. Por se tratar de um instrumento de campo, uma atenção especial foi dedicada aos aspectos de consumo e estabilidade térmica de seus circuitos.

\section{O Instrumento}

A figura 2 mostra o diagrama em blocos simplificado do instrumento construído no LDSM/ON. Um microcontrolador dedicado produz sinais de excitação e detecção com frequências de $6 \mathrm{kHz}$ e $12 \mathrm{kHz}$, respectivamente (oscillator and phase generator).

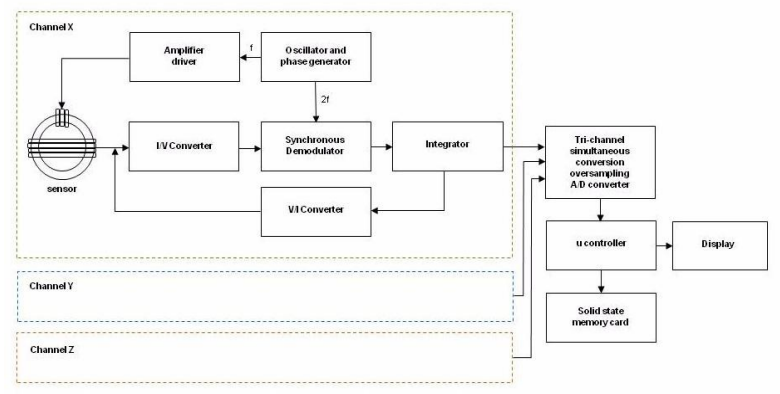

Figura 2 - Diagrama em blocos do equipamento

O sinal de saída $\boldsymbol{f}$, com frequência de $6.5 \mathrm{kHz}$, é em seguida amplificado (Amplifier driver), levando o núcleo amorfo (sensor) à saturação. O sinal produzido pela interação entre o campo local e a saturação do núcleo induz em uma bobina detectora enrolada sobre o sensor um sinal de amplo espectro com harmônicos pares, cujas amplitudes são proporcionais à densidade de fluxo do campo a ser medido. Uma chave analógica comandada pelo sinal $2 \boldsymbol{f}$, de $13 \mathrm{kHz}$, atua como demodulador síncrono, retificando o sinal pulsado produzido pela bobina sensora. Por meio de ajustes no código embarcado no chip micro controlador, a fase relativa entre os sinais $f$ e $\mathbf{2} f$ é controlada de forma a se obter uma demodulação síncrona eficiente. Um estagio integrador filtra o sinal retificado, produzindo um nível DC de voltagem a ser convertida para leitura por um conversor analógico digital de alta resolução. A voltagem assim obtida é realimentada para a bobina detectora, por meio de um conversor V/I, produzindo um efeito linearizador e estabilizador de ganho do circuito, contribuindo com sua precisão e reduzindo o ruído produzido pelos circuitos auxiliares.

Um microcontrolador programado em linguagem " $\mathrm{C}$ " controla um conversor analógico/digital de três canais com 22 bits de resolução, efetuando o cálculo de médias, filtragens e escalas, traduzindo os sinais analógicos em sentenças digitais que são em seguida transmitidas para um display de cristal líquido e para uma porta de comunicação para conexão com um computador portátil. 


\section{Resultados}

Após a construção do instrumento, foram realizados testes em laboratório para verificação de seus parâmetros principais. O ruído e deriva do sensor foram analisados com o auxílio de uma blindagem magnética de cinco camadas, construída em liga de alta permeabilidade (mu-metal) e de um analisador de espectro digital de alta resolução.

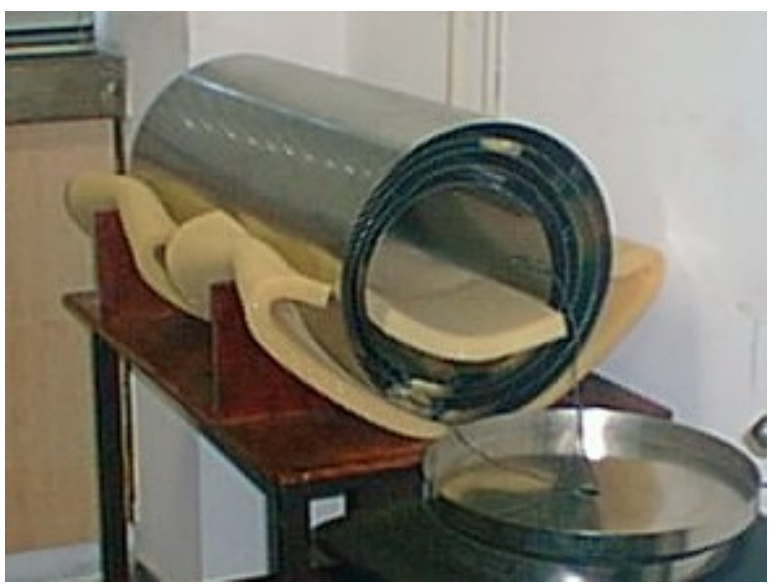

Figura 3 - Blindagem magnética em mu-metal de cinci camadas do LDSM/ON, utilizada para avaliação de ruído próprio e deriva de sensores magnéticos.

O procedimento de calibração foi realizado por meio da produção de um campo magnético sintético, controlado através de uma bobina de Helmholtz triaxial e de fontes geradoras de corrente de precisão.
Conforme apresentado na figura 5, o magnetômetro apresentou uma ótima linearidade, com desvio da reta da ordem de $2.10^{-4}$. em uma faixa de até \pm 100 uT -, duas vezes aquela planejada para o instrumento.

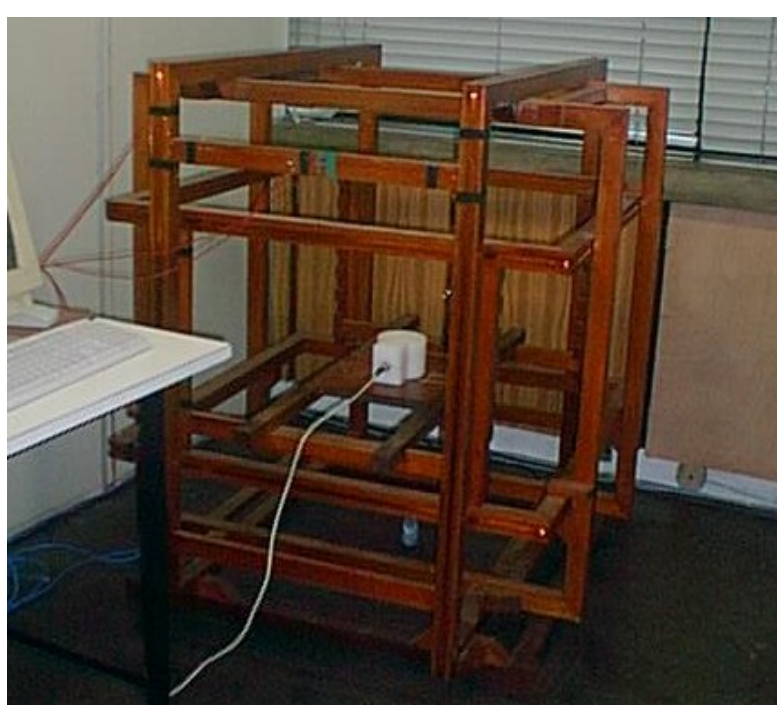

Figura 4 - Testes com o sensor amorfo em bobina de Helmoltz.

Com o elemento sensor isolado pela blindagem magnética, após um período de integração de uma hora, foi obtido um ruído médio inferior a $0.01 \mathrm{nT}$ em toda a faixa de frequências até $2 \mathrm{~Hz}$ (figura 6), somando-se o ruído próprio do sensor ao ruído produzido pelos circuitos eletrônicos.

Sensor Amorfo

Teste de linearidade em bobina de Helmholtz

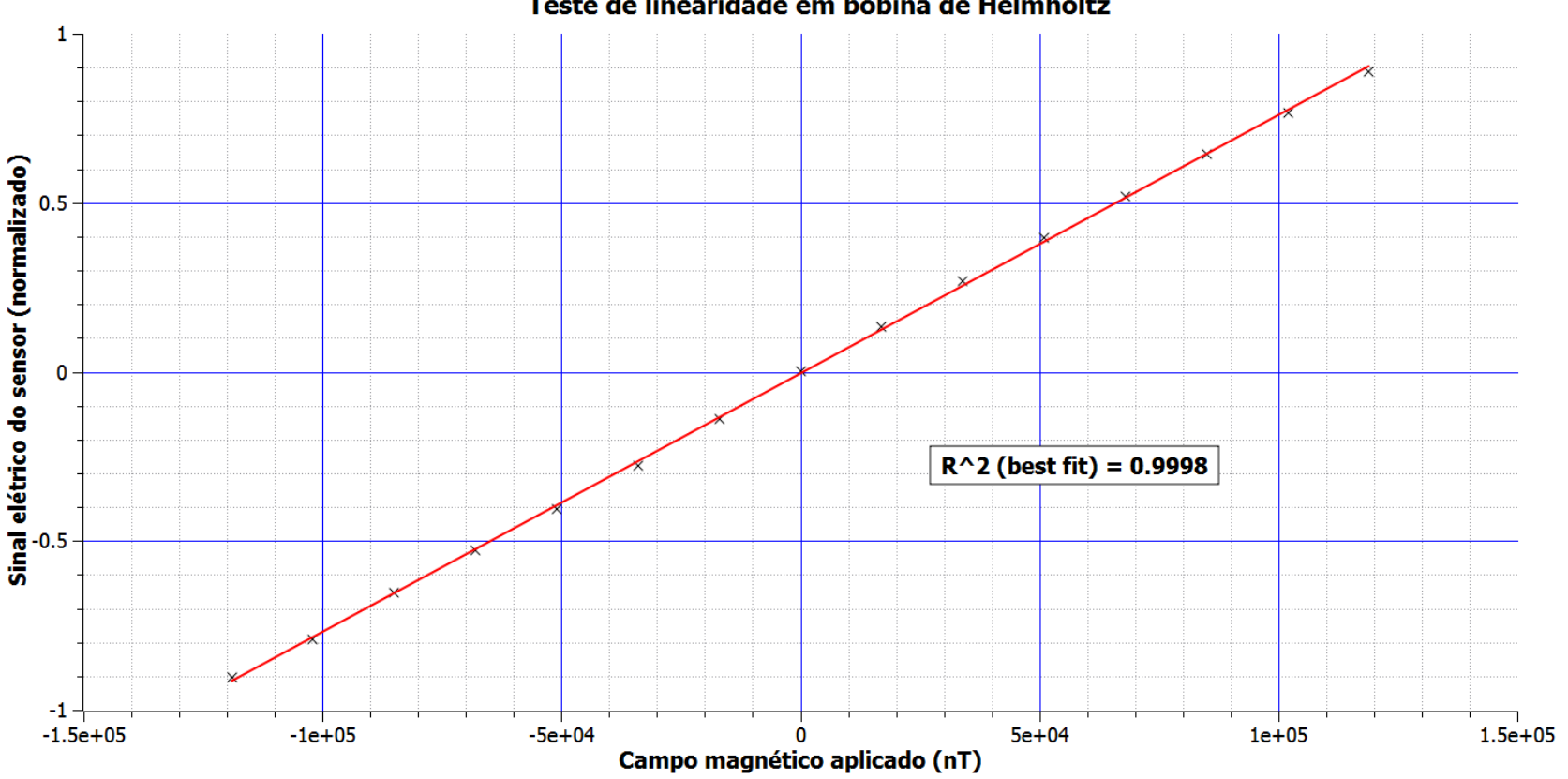

Figura 5 - Calibaração e levantamento da linearidade do instrumento obtidos com o uso da bobina de Helmoltz. 


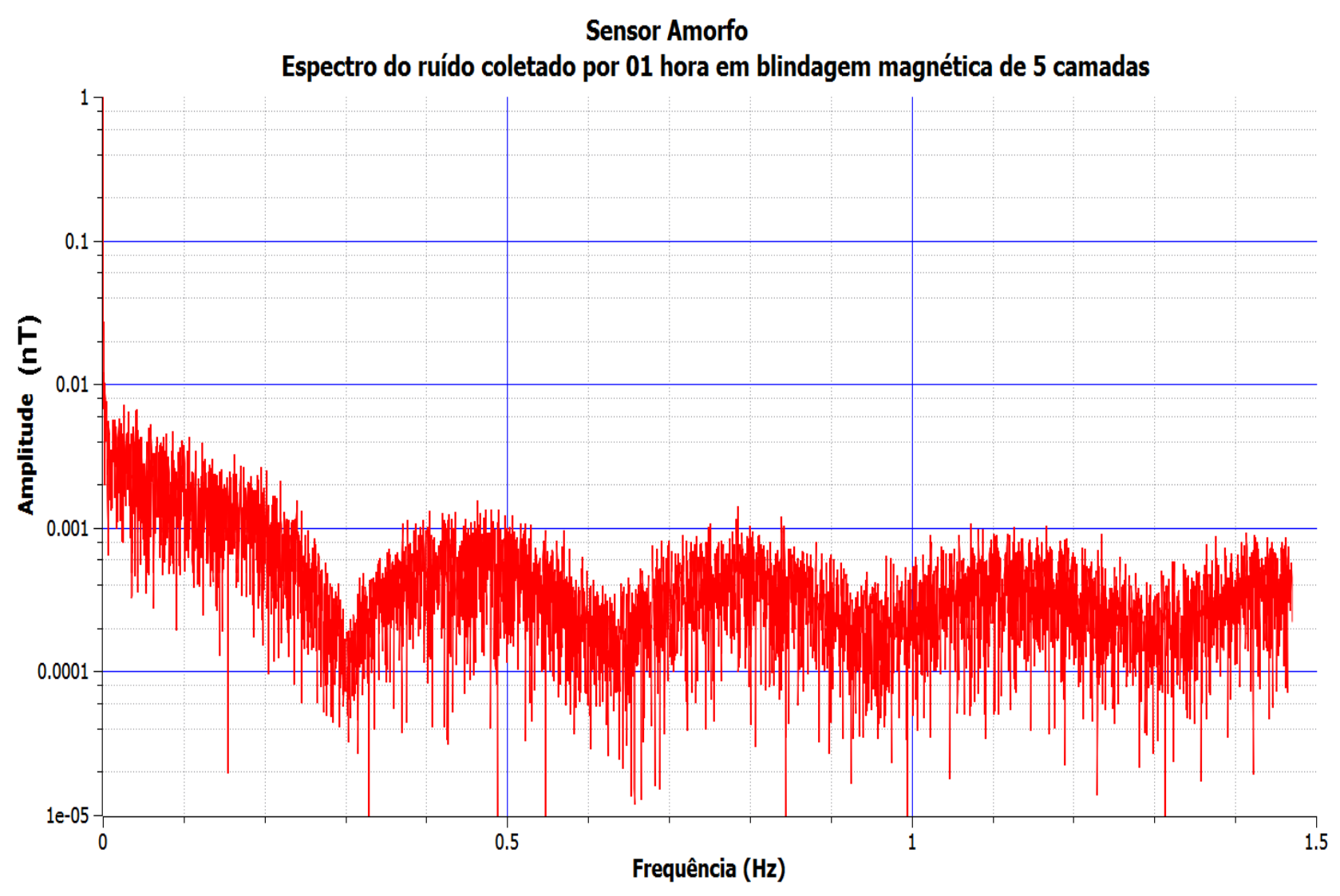

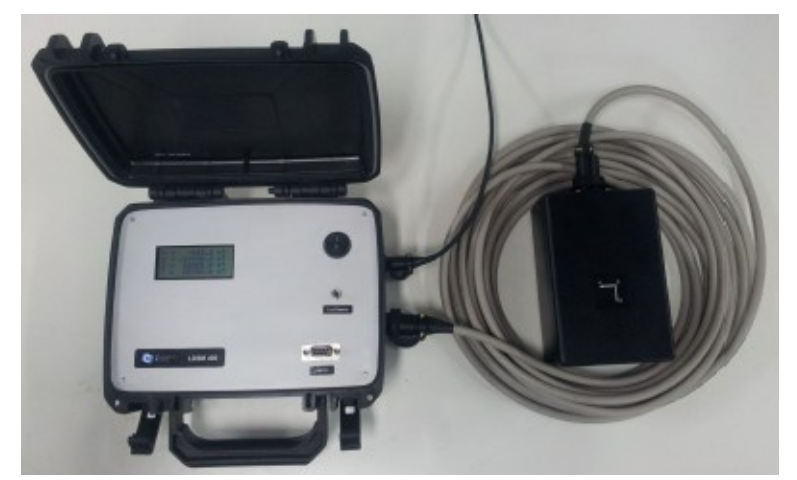

Figura 7 - Aspecto final do instrumento construído.

\section{Discussão e Conclusões}

O instrumento construído apresentou excelente performance, compatível com o seus objetivos. Após os testes e calibrações, a primeira unidade produzida deste magnetômetro foi fornecida para uma empresa de prospecção geológica. Este equipamento encontrase agora em uso no campo, sendo utilizado para a localização e mapeamento de jazidas minerais.

O LDSM/ON iniciou uma nova etapa de desenvolvimento, para a inclusão de recursos avançados a este magnetômetro de prospecção, incluindo a integração com antenas de GPS e sensores de horizonte artificial (nível eletrônico), visando uma utilização mais prática e eficiente.

\section{Agradecimentos \\ À FAPERJ pelo auxílio concedido}

\section{Referências}

Benyosef, Luiz Carlos de Carvalho . Development of low noise fluxgate sensors. Geofísica Internacional, México, v. 48, n.01, p. 111-120, 1998.

Benyosef, Luiz Carlos de Carvalho ; NOVAK, M. A. . Magnetômetro de Núcleos Saturados. Revista de Física Aplicada e Instrumentação, São Paulo, v. 11, n.03, p. 131-137, 1996.

Jankowski, J., Sucksdorff, C., Guide for Magnetic Measurements and Observatory Practice, 1 ed. Warsaw, NOAA / IAGA, 1996.

Benyosef, Luiz Carlos de Carvalho ; Joel R. Teodosio ; Vladimir E. Taranichev ; Boris V. Zhalnin . Improvements on CoFeSiB Amorphous Ribbon for Fluxgate Sensor Cores. Scripta Metallurgica et Materialia, v. 33, n.9, p. 1451-1454, 1995.

Primdahl, F., Temperature Compensation of Fluxgate Magnetometers, IEEE Trans. on Magnetics, vol Mag.5 1989.

Primdahl, F., The Fluxgate Magnetometer, J. Phys. Sci. Instrum., vol 12, 1979. 